\title{
Insertion Experiment and Resistance Tests of Solid Polymer Micro Needles Array
}

\author{
Samir S. Abubaker, Zhang Yajun, Jin Zhiming \\ College of Mechanical and Electrical Engineering, Beijing University of Chemical Technology, \\ Beijing, China. \\ Samir@mail.buct.edu.cn, zhyj@mail.buct.edu.cn,jinzm@mail.buct.edu.cn*
}

\begin{abstract}
Keywords: Polymer micro-needle, Hot embossing, Taguchi method.
Abstract. Microneedles have been made from silicon, glass, and metal. Nowadays, many promising technologies are focused on polymers since they are biodegradable, biocompatible, and easy to fabricate. They can penetrate safely in the body, providing a drug distribution method and eliminating the risk of the needles fracturing and buckling. The problem faced by most transdermal patches is the low permeability of skin. Microneedles have been shown to greatly increase the skin's permeability, allowing for an effective transfer of drugs. They however still face numerous challenges. This work addresses the challenge to effectively microneedle insertion into porcine skin, which is similar to human skin. A 7x7 solid polymer microneedle array made by hot embossing process was tested for insertion. Results show that the array is extremely resistant to insertion, it can withstand very high forces and even multiple insertions without blunting.
\end{abstract}

\section{Introduction}

With the development of micro-electro-mechanical systems (MEMS) and materials science, a more efficient and safer traditional transdermal drug delivery (TDD) like microneedle (MN) emerges. MN technology has received much attention in the medical community and the beauty industry. Microneedles (MNs) can eliminate the side effects of typical injection needle, such as skin damage, infection, and pain, and easy to use, in contrast to hypodermic needles [1]. Furthermore as an alternative, transdermal patches provide convenient, time-release delivery that avoids the gastrointestinal tract [2]. Appeared clearly for MNs technology development, it becomes possible for MNs array to strengthen the TDD, in addition to the increase of the skin permeability, therefore to deliver drugs into skin, such as insulin and vaccine, providing a new direction for drug delivery systems. MNs will play a decisive role in promoting micro-sample analysis, trace injection, blood fluid analysis, sampling and vaccines against influenza [3]. MNs have been demonstrated to be pain-free and potentially low-cost and easy-to use [4]. Generally, MNs should not break when bent slightly, and not rupture when pulled out after piercing the skin (except dissolvable MNs).

In this paper, solid polymer MNs manufactured by hot embossing method was discussed, a mechanical model of MN was established, and then an appropriate force equations was built. After the strength of a polymer MN was verified, the piercing situations of different shapes were simulated, the optimized structures of MNs were obtained. Based on the stress condition, three kinds of common polymers with different geometries were simulated using MATLAB and ANSYS. The results showed that all kinds could satisfy the strength requirements.

Based on that, PMMA, PC and PLA were chosen to fabricate MNs, details of MNs fabrication with hot embossing process were presented [5]. To minimize the cycle time while maintaining quality, non-isothermally of embossing step was used [6]. Through the experiments, the significant embossing factors were decided by Taguchi method, and quality of MNs are evaluated. Lastly, tests were made to verify the properties of MN. 


\section{Experimental procedure}

This part presents an illustrative sequence of implementation of experimental work for process parameters optimization under three parameter settings and one-quality response. Taguchi orthogonal method is used for investigating the optimal performance characteristics from a set of factors through design of experiment (DOE). Hot embossing process has many potential factors, although all of these machine settings are acceptable factors, DOE aims to reduce the number of these factors thus reduce the cycles to be run. Based on Taguchi orthogonal method to design experiment, the array $L 16\left(4^{3}\right)$ was selected in this paper. The control factors and their levels are shown in Table 1 below (eg.PMMA). There are 16 treatments for all combinations; tow replications are used for each setting to increase the sensitivity of statistical analysis.

Table 1. The specific process parameters with their levels(PMMA)

\section{Parameters}

Embossing temperature $\left({ }^{\circ} \mathrm{C}\right)$

Embossing pressure (MPa)

Embossing time (s)

\begin{tabular}{cccc}
\multicolumn{4}{c}{ Levels } \\
\hline 1 & 2 & 3 & 4 \\
120 & 125 & 130 & 135 \\
5 & 7 & 9 & 11 \\
100 & 150 & 200 & 250
\end{tabular}

\section{Experimental and Measurements machine used}

The hot embossing apparatus is a flat-platen thermal embossing apparatus developed by the Institute of Plastics and Plastic Engineering of Beijing University of Chemical Technology as shown in Fig.1 (a). The effective area of embossing is $160 \times 80 \mathrm{~mm}$, a PLC controls the servomotor movement, the servo motor drives the screw lift to drive the upper and lower platen movement. The speed of the pressing upper mold platen could be set from 0.5 to $5.0 \mathrm{~mm} / \mathrm{s}$. The pressing force of embossing can be acquired up to $50 \mathrm{kN}$ with a control accuracy of $\pm 50 \mathrm{~N}$. A mold temperature controller from room temperature to $170{ }^{\circ} \mathrm{C}$ can control the temperature of the mold with a control accuracy of $\pm 1^{\circ} \mathrm{C}$.

The JTVMS-1510T 3D Image Measurement System Fig.1(b), product of China Dongguan Jaten Precision Instrument, carried on characterization of the microstructure of the embossed substrates.

The experimental mold was made of stainless steel with the size of $20 \times 20 \mathrm{~mm}$ and a thickness of $2 \mathrm{~mm}$, and assumed isotropic and elastic because it involves little deformation as compared to the polymer. As shown in Fig.1(c), the mold is distributed with $7 \times 7$ MNs concave conical array, the distance between the adjacent cone hole is $2 \mathrm{~mm}$, the $\mathrm{MN}$ counterbore bottom diameter (cone Platform diameter) is $250 \mu \mathrm{m}$, the top diameter (truncated cone) is $50 \mu \mathrm{m}$, and the depth is about $500 \mu \mathrm{m}$.

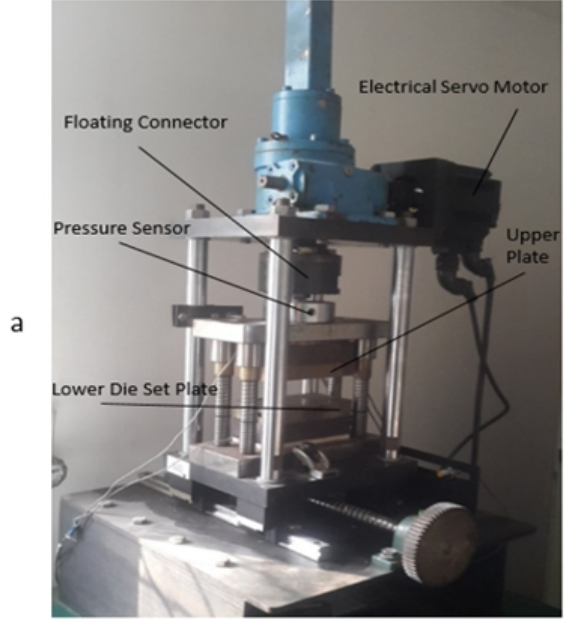

(a) Hot embossing machine b

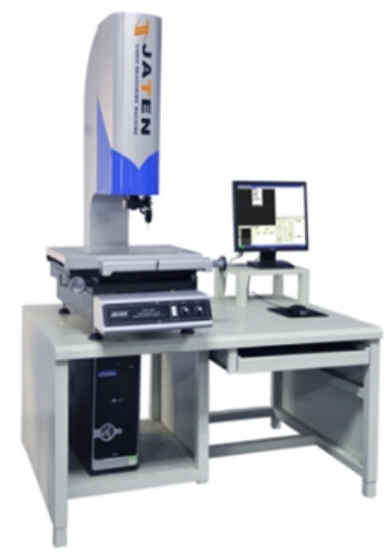

(b)Video measurement

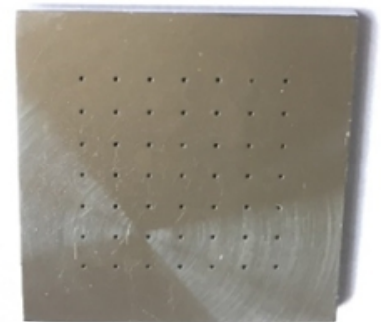

(c)Microneedle mold

Fig.1. Equipment for experiments 


\section{Materials and methods}

The polymer materials PMMA, PC, and PLA with the size of $24 \times 24$ and $1 \mathrm{~mm}$ in thickness were selected as the substrate material of the MNs. Main properties of the materials were listed in Table 2.

Table 2 Main properties of polymers

\begin{tabular}{|l|c|c|c|c|c|}
\hline Material & $\begin{array}{c}\text { Young's } \\
\text { modulus(GPa) }\end{array}$ & $\begin{array}{c}\text { Poisson's } \\
\text { ratio }\end{array}$ & $\begin{array}{c}\text { Tensile } \\
\text { strength }(\mathrm{MPa})\end{array}$ & $\begin{array}{c}\text { Density } \\
\left(\mathrm{g} / \mathrm{cm}^{2}\right)\end{array}$ & $\begin{array}{c}\text { Glass-transition } \\
\text { temperature }\left({ }^{\circ} \mathrm{C}\right)\end{array}$ \\
\hline PC & $2.0-2.4$ & $0.37-0.39$ & $55-75$ & $1.2-1.22$ & 145 \\
\hline PMMA & $1.8-3.1$ & $0.35-0.4$ & $50-77$ & $1.17-1.20$ & 105 \\
\hline PLA & $3-4$ & 0.36 & $40-60$ & $1.21-1.43$ & 65 \\
\hline
\end{tabular}

\section{The hot embossing Cycle}

The experiment cycle starts when the mold is placed on the lower set platen, and heated above its glass transition temperature $\left(T_{g}\right)$ to the desired embossing temperature as illustrated in Fig.2. After reaching the embossing temperature, the polymer substrate was placed at the top of mold between the platens, the upper platen is then driven to the lower platen at $\approx 3 \mathrm{~mm} / \mathrm{s}$ until a threshold force is detected, indicating that the upper platen is in contact with substrate and the mold. The upper platen then was driven down at $\approx 0.5 \mathrm{~mm} / \mathrm{s}$ pressing the substrate until the desired embossing pressure is reached. The embossing pressure was maintained for the desired embossing time. Then the platens were cooled to the demolding temperature, while the pressure is still maintained, the platens are separated after the Demolding Temperature is reached. The mold with substrate are taken out from the machine, the part is separated from the mold using tweezer and quenched in water, then the mold placed again on the lower die set platen with the demolding temperature and start with the second test.

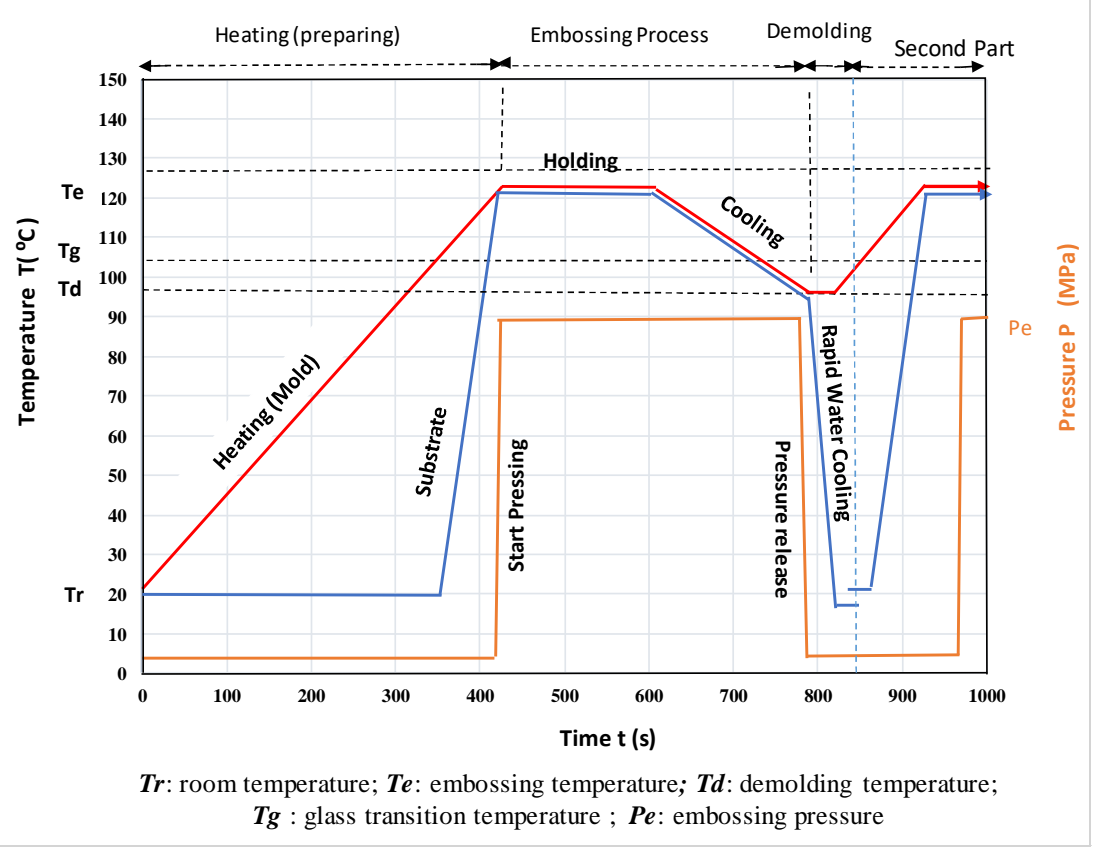

Fig. 2. Illustrative Diagram for Hot Embossing Process

\section{Skin Penteration Experimental results and analysis of MNs}

Porcine skin was cut to four pieces 50x50mm and marked as number 0, 1, 2, 3, 4. First, Measure the skin moisture for all parts before acupuncture and record it, then using the universal testing machine. For pressing the skin parts with MNs. No. 0 no pressing part, then pressing the parts 1,2 , and 3 with 
$50,60,70$, and(70N twice with different direction) respectively, with 3 minutes holding time. The moisturizer was applied on the four skin parts as shown in Fig.3, and rubbed for two minutes for all parts. Then stored in fresh keeping bags to prevent air-drying. After 10 minutes, the surface of the skin was cleaned with a paper towel to dry the surface moisture and oil content.

Then all parts were measured (ambient temperature $21^{\circ} \mathrm{C}$, humidity $33 \% \mathrm{RH}$ ). Experimental data is shown in Table 3 and Fig. 4 below.

Table 3 Test data of experiment

\begin{tabular}{|c|c|c|c|c|c|c|}
\hline \multirow{2}{*}{ Category } & \multirow{2}{*}{ Type } & \multicolumn{5}{|c|}{ Test parts } \\
\cline { 2 - 6 } & & untreated & 1 & 2 & 3 & 4 Twice \\
\hline \multirow{2}{*}{$\begin{array}{c}\text { Before test no } \\
\text { moisturizer }\end{array}$} & Moisture & 87.6 & 87.6 & 88.0 & 87.7 & 87.7 \\
\cline { 2 - 7 } After 15 min & Oil & 27.3 & 28.0 & 28.1 & 27.7 & 27.6 \\
\cline { 2 - 7 } & Moisture & 90.2 & 89.6 & 89.6 & 89.6 & 92.0 \\
\hline \multirow{2}{*}{ After 30 min } & Oil & 28.8 & 28.6 & 28.6 & 28.8 & 28.9 \\
\cline { 2 - 7 } & Ooisture & 90.6 & 90.1 & 91.1 & 91.1 & 92.1 \\
\hline \multirow{2}{*}{ After 60 min } & Moisture & 28.8 & 28.8 & 29.1 & 29.1 & 29.3 \\
\cline { 2 - 7 } & Oil & 28.8 & 28.9 & 29.0 & 29.1 & 29.5 \\
\hline \multirow{2}{*}{ After120 min } & Moisture & 90.0 & 91.0 & 90.4 & 91.7 & 92.3 \\
\cline { 2 - 7 } & Oil & 28.8 & 29.1 & 28.9 & 29.3 & 29.7 \\
\hline \multirow{2}{*}{ After 4 hours } & Moisture & 88.5 & 91.1 & 91.1 & 91.3 & 92.0 \\
\cline { 2 - 7 } & Oil & 28.3 & 29.3 & 29.1 & 29.2 & 29.9 \\
\hline
\end{tabular}

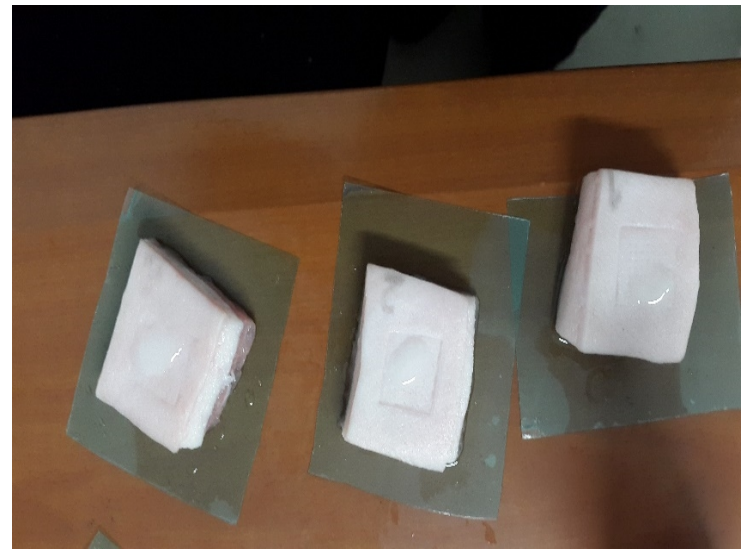

Fig.3. Porcine skin treatment with moisturizer

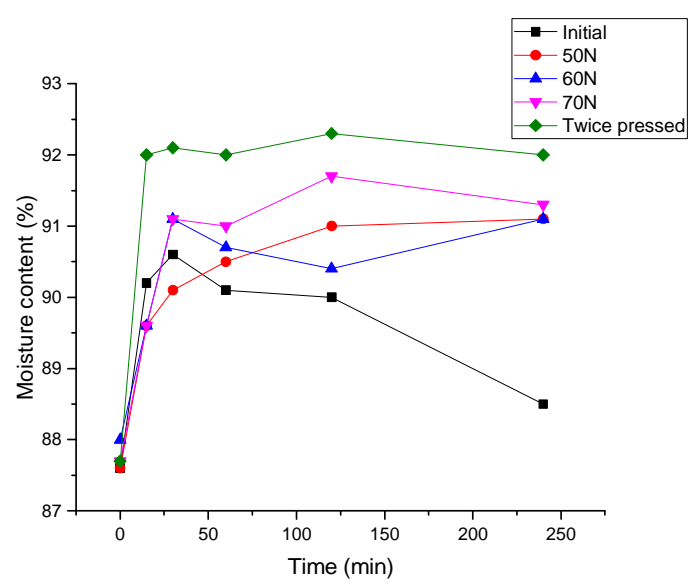

Fig. 4. Resultant curves of porcine skin moisture

\section{Mechanical Strength and Rupture of MNs}

In this test, Polyethylene (PE) film of a thickness $60 \mu \mathrm{m}$ folded into 7 layers pressed against the MNs. The MNs are inserted into the PE film layers supported by $2 \mathrm{~mm}$ thickness Styrene Ethylene butylene Styrene (SEBS) plate, with a force of $10 \mathrm{~N}, 20 \mathrm{~N}, 25 \mathrm{~N}, 30 \mathrm{~N}, 50 \mathrm{~N}$ and held for $15 \mathrm{~s}$, observation made after each insertion shows that the maximum depth value reached by forces above $25 \mathrm{~N}$ based on the 6 layers pierced as shown in Fig.5. Moreover, the stratum corneoum (SC) is the main obstacle with around $40 \mu \mathrm{m}$ thickness therefore, once the microneedles have penetrated the SC, the resistive force falls drastically. In addition, no remarkable damage observed after repeatable insertion of MNs. 

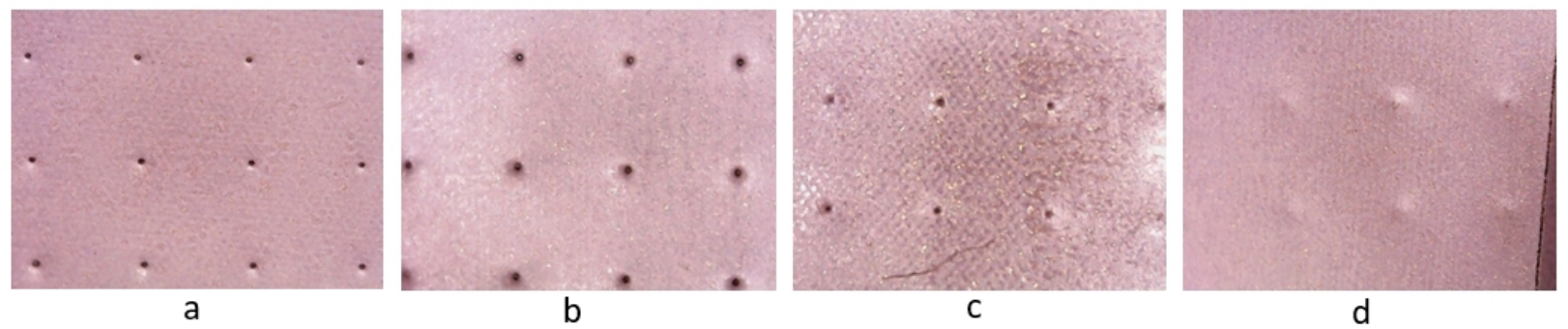

Figure 5. PE film layers after MNs pressing with F=30N (a) $1^{\text {st }}$ Layer, (b) $2^{\text {nd }}$ Layer, (c) $6^{\text {th }}$ Layer, (d) $7^{\text {th }}$ Layer

\section{Conclusion}

The optimized solid polymer MN for cosmetic purpose was fabricated with low cost because of its innovative fabrication method that implies hot embossing. The biocompatible polymeric materials not only insure excellent biocompatibility for the patients but also extremely resistant considering insertion into the skin. It can withstand very high forces and does not break but crushes making the $\mathrm{MN}$ very safe for the patients as they cannot be broken and stay into the skin. Different insertion tests were performed on the porcine skin and PE film (which shows excellent resemblance to porcine skin). Results demonstrate that the insertion force is the most important parameter but can be compensated by optimising insertion speed and holding time. While the insertion force for our MN is already largely acceptable regarding patient comfort. All polymer materials tested assure good penetration, and no buckling and damage, and can be used safely.

\section{References}

[1] Prausnitz, Mark R., and Robert Langer. "Transdermal drug delivery." Nature biotechnology 26, no. 11 (2008): 1261.

[2] Prausnitz, Mark R. "Analysis: overcoming skin's barrier: the search for effective and user-friendly drug delivery." Diabetes technology \& therapeutics 3, no. 2 (2001): 233-236.

[3] Sullivan, Sean P., Dimitrios G. Koutsonanos, Maria del Pilar Martin, Jeong Woo Lee, Vladimir Zarnitsyn, Seong-O. Choi, Niren Murthy, Richard W. Compans, Ioanna Skountzou.

[4] El-Laboudi, Ahmed, Nick S. Oliver, Anthony Cass, and Desmond Johnston. "Use of microneedle array devices for continuous glucose monitoring: a review." Diabetes technology \& therapeutics 15, no. 1 (2013): 101-115.

[5] Worgull, M., J-F. Hétu, K. K. Kabanemi, and M. Heckele. "Hot embossing of microstructures: characterization of friction during demolding." Microsystem Technologies 14, no. 6 (2008): 767-773.

[6] Wu Daming, Jingyao Sun, Ying Liu, Zhenzhou Yang, Hong Xu, Xiuting Zheng, and Pengsheng Gou. "Rapid fabrication of microstructure on PMMA substrate by the plate to plate Transition-Spanning isothermal hot embossing method nearby glass transition. 